\title{
Dielectric boundary effects on the interaction between planar charged surfaces with counterions only
}

Cite as: J. Chem. Phys. 148, 164103 (2018); https://doi.org/10.1063/1.5022226

Submitted: 12 January 2018 . Accepted: 05 April 2018 . Published Online: 25 April 2018

Alexandre P. dos Santos (iD, and Roland R. Netz (D)
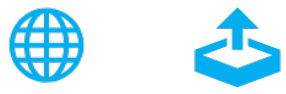

\section{ARTICLES YOU MAY BE INTERESTED IN}

Kinetic energy definition in velocity Verlet integration for accurate pressure evaluation The Journal of Chemical Physics 148, 164109 (2018); https://doi.org/10.1063/1.5008438

Simulations of Coulomb systems confined by polarizable surfaces using periodic Green functions

The Journal of Chemical Physics 147, 184105 (2017); https://doi.org/10.1063/1.4997420

Focus Article: Oscillatory and long-range monotonic exponential decays of electrostatic interactions in ionic liquids and other electrolytes: The significance of dielectric permittivity and renormalized charges

The Journal of Chemical Physics 148, 193701 (2018); https://doi.org/10.1063/1.5010024

Where in the world is AIP Publishing? Find out where we are exhibiting next 


\title{
Dielectric boundary effects on the interaction between planar charged surfaces with counterions only
}

\author{
Alexandre P. dos Santos ${ }^{1,2, a)}$ and Roland R. Netz ${ }^{2, b)}$ \\ ${ }^{1}$ Instituto de Física, Universidade Federal do Rio Grande do Sul, Caixa Postal 15051, \\ CEP 91501-970 Porto Alegre, RS, Brazil \\ ${ }^{2}$ Fachbereich Physik, Freie Universität Berlin, 14195 Berlin, Germany
}

(Received 12 January 2018; accepted 5 April 2018; published online 25 April 2018)

\begin{abstract}
Using Monte Carlo simulations in conjunction with periodic Green's function methods, we study the interaction between planar charged surfaces with point-like counterions only in the presence of dielectric boundaries. Based on the calculated pressure profiles, we derive phase diagrams featuring correlation-induced negative pressure and thus attraction between the plates for large coupling parameters, i.e., low temperature or high surface charge and high ion valency. The counterion density profiles for low-dielectric and high-dielectric (metallic) surfaces are very different from the idealized case of a homogeneous dielectric constant. By contrast, the phase diagrams including the critical point and the two-phase coexistence region are rather insensitive to the presence of dielectric boundary effects. The single-image approximation that has been used in simulations before is by comparison with the exact formalism shown to be very accurate for low-dielectric surfaces but not for metallic surfaces. Published by AIP Publishing. https://doi.org/10.1063/1.5022226
\end{abstract}

\section{INTRODUCTION}

The design of synthetic surfaces with tailored interaction properties when dissolved in aqueous solution is of fundamental importance for many applications, ${ }^{1-5}$ for example, in drug delivery scenarios ${ }^{6-9}$ or in biosensor technology. ${ }^{9,10}$ In all these situations, it is important to understand the interaction between such surfaces. ${ }^{7,11-17}$ The Derjaguin-LandauVerwey-Overbeek (DLVO) theory is a very useful model that describes the interaction between charged surfaces as the sum of hydration repulsion and electrostatic pressure. ${ }^{18-20}$ Atomistic simulations have recently shed light on the hydration pressure between membranes. ${ }^{21,22}$ The electrostatic contribution to the overall interaction pressure is typically described on the mean-field level by the Poisson-Boltzmann (PB) theory. However, the standard PB theory does now allow accounting for phenomena encountered for highly charged surfaces such as charge reversal ${ }^{23,24}$ or ion specific effects. ${ }^{25-27} \mathrm{New}$ theoretical approaches go beyond the mean-field approximation. ${ }^{28-36}$ For example, integral equations have been used for the calculation of the interaction between highly charged surfaces that are embedded in electrolyte solution. ${ }^{28}$ Field-theory methods have been applied in the limit of strong electrostatic coupling and showed that highly charged surfaces attract each other at low temperatures and/or high counterion valency. ${ }^{30-32}$ The introduction of dielectric boundaries at charged surfaces increases the complexity of the problem. In fact, the mean-field theory for planar surfaces is not modified by the presence of dielectric boundaries and thus gives no information on dielectric effects in planar systems. By contrast, some approaches based on liquid theory and variational treatments allowed

a)Electronic mail: alexandre.pereira@ufrgs.br

b)Electronic mail: rnetz@physik.fu-berlin.de considering dielectric effects. ${ }^{37-39}$ Various simulation methods were developed in order to properly take into account dielectric boundaries. ${ }^{40-45}$ In the present paper, we use Monte Carlo (MC) simulations to study the interaction between uniformly charged surfaces with counterions only in the presence of a dielectric contrast. The dielectric boundary at the planar charged surfaces is handled with a recently introduced method based on periodic Green's functions. ${ }^{46}$ In order to regularize the Hamiltonian, we introduce a distance of closest approach between the counterions and the dielectric boundaries. This distance can be interpreted as being due to hydration effects which give rise to an effective repulsion between an ion and a charged surface, we therefore call this separation the hydration length. The counterion density profiles are shown to depend sensitively on the ionic hydration length and on the dielectric contrast. However, the pressure curves, in particular, around the critical point obtained for large coupling parameters, are much less sensitive to the values of the dielectric contrast and the hydration length. We also show that the single-image approximation, which has been employed in simulations before to account for dielectric boundary effects, is a very good approximation for low-dielectric surfaces. ${ }^{32}$

The manuscript is organized as follows. In Sec. II, the model, the simulation method, and the definitions used are presented. Afterwards counterion density profiles, pressure profiles, and phase diagrams featuring the two-phase coexistence region and the critical point are presented. In Sec. IV, conclusions and future perspectives are outlined.

\section{MODEL AND SIMULATION METHOD}

Our simulation system is represented in Fig. 1. We consider $N=100$ counterions of charge $q e$, where $q$ is the valency 


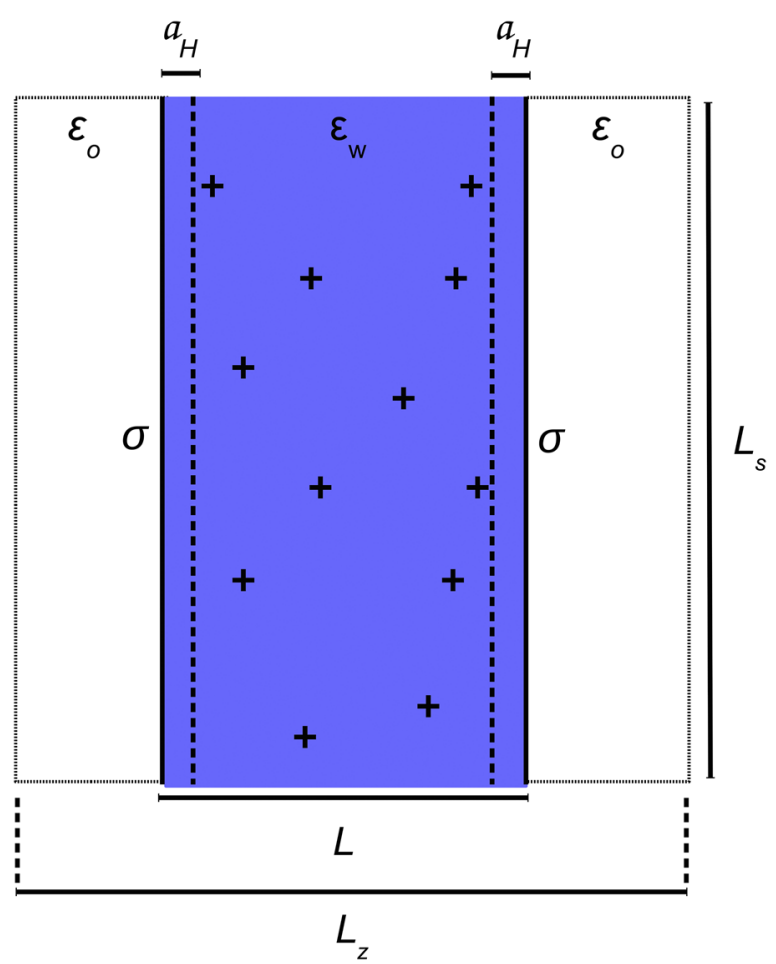

FIG. 1. Schematic representation of the system. Point-like counterions are confined between surfaces with uniform charge densities $\sigma$ separated by the distance $L$. The ions are excluded by the distance $a_{H}$ from both surfaces. The uniform dielectric constant between the surfaces is $\epsilon_{w}$, while outside it is $\epsilon_{o}$.

and $e$ is the elementary charge. They are confined in the region $-L_{s} / 2<x<L_{s} / 2,-L_{s} / 2<y<L_{s} / 2$, and $a_{H}<z<L-a_{H}$, where $L$ is the distance between the charged planar surfaces, on which the dielectric boundaries are located, and $a_{H}$ denotes the hydration length. By construction, the counterions are not allowed to approach the surfaces by a distance less than $a_{H}$. The periodic simulation box has dimensions $L_{s}, L_{s}$, and $L_{z}=3 L_{s}$. The vertical box size $L_{z}$ length is larger than the surface separation $L$ in order to allow for Ewald summation techniques including corrections for the slab geometry. ${ }^{47}$ The coupling parameter is defined as $\Xi=q^{2} \lambda_{B} / \mu$, where $\lambda_{B}$ $=e^{2} / k_{B} T \epsilon_{w}$ is the Bjerrum length, while $\epsilon_{w}$ is the uniform water dielectric constant in between the surfaces. For small values of $\Xi$ and uniform dielectric constants, the PB theory is valid, while for large values of $\Xi$ the PB theory breaks down and the strong-coupling theory becomes valid. ${ }^{33}$ In the presence of dielectric contrast, the PB equation has been shown to be no longer valid even for weak coupling. ${ }^{48}$ The GouyChapman length is given by $\mu=1 /\left(2 \pi q \lambda_{B} \sigma\right)$, where $\sigma$ is the charge density of both surfaces. Outside the confining surfaces, the dielectric constant is $\epsilon_{o}$. The dielectric contrast $\gamma$ is defined as

$$
\gamma=\frac{\epsilon_{w}-\epsilon_{o}}{\epsilon_{w}+\epsilon_{o}} .
$$

The minimal set of parameters that describes our system consists of the coupling parameter $\Xi$, the dielectric contrast $\gamma$, the rescaled distance between the surfaces, defined as

$$
\bar{L}=\frac{\left(L-2 a_{H}\right)}{\mu},
$$

and the rescaled hydration length

$$
\bar{a}_{H}=\frac{a_{H}}{\mu \Xi^{1 / 2}} .
$$

The MC simulations are performed in the NVT ensemble. The counterions perform isotropic random short moves with step sizes adjusted in order to obtain an acceptance ratio around $50 \%$. Also long moves to an arbitrary position inside the simulation box are performed. The ratio of long and short moves is set to unity. The system is equilibrated for $10^{5} \mathrm{MC}$ steps, where one MC step is defined as one move attempt per particle on average. In the production run, $10^{6}$ counterion configurations are saved for further analysis every $100 \mathrm{MC}$ steps. The total system energy is given by

$$
U=U_{E w}+U_{p},
$$

where $U_{E w}$ is the energy in the absence of dielectric boundaries. It is calculated by the modified 3D Ewald method for non-neutral systems and slab geometry. ${ }^{47}$ The energy term $U_{p}$ takes care of all additional terms due to the dielectric boundaries and is calculated using periodic Green's functions. ${ }^{46} \mathrm{It}$ can be written as

$$
\begin{aligned}
\beta U_{p}= & \beta U_{m e}+\lambda_{B} \sum_{\mathbf{k}}^{\prime} \frac{\pi \gamma}{L_{S}^{2}|\mathbf{k}|\left[1-\gamma^{2} \exp (-2|\mathbf{k}| L)\right]} \\
& \times\left\{f_{1}(\mathbf{k})^{2}+f_{2}(\mathbf{k})^{2}+\exp (-2|\mathbf{k}| L)\left[f_{3}(\mathbf{k})^{2}\right.\right. \\
& \left.\left.+f_{4}(\mathbf{k})^{2}+2 \gamma f_{2}(\mathbf{k}) f_{4}(\mathbf{k})+2 \gamma f_{3}(\mathbf{k}) f_{1}(\mathbf{k})\right]\right\},
\end{aligned}
$$

where $\mathbf{k}=\left(2 \pi n_{x} / L_{s}, 2 \pi n_{y} / L_{s}\right)$ is the reciprocal vector and $n_{x}$ and $n_{y}$ are integers. The prime over the summation symbol excludes the term $\mathbf{k}=(0,0)$ from the summation. The number of $\mathbf{k}$ vectors used in the summation must increase with the lateral size $L_{s}$ and with the proximity of the ion to the surfaces. The term $U_{m e}$ is considered only for metallic surfaces $(\gamma=-1)$ and corresponds to the divergent term $\mathbf{k}=(0,0)$ which is considered separately. ${ }^{46}$ It is given by

$$
\beta U_{m e}=-\frac{2 \pi \lambda_{B}\left(\sum_{i=1}^{N} q_{i} z_{i}+\sigma L_{s}^{2} L\right)^{2}}{L_{s}^{2} L},
$$

where $N$ is the number of counterions and $\left(x_{i}, y_{i}, z_{i}\right)$ is the counterion position with charge $q_{i}$. The charge of the surfaces inside the main simulation box is included. The functions are given by

$$
\begin{aligned}
& f_{1}(\mathbf{k})=\sum_{i=1}^{N} q_{i} \cos \left(k_{x} x_{i}+k_{y} y_{i}\right) \exp \left(-|\mathbf{k}| z_{i}\right), \\
& f_{2}(\mathbf{k})=\sum_{i=1}^{N} q_{i} \sin \left(k_{x} x_{i}+k_{y} y_{i}\right) \exp \left(-|\mathbf{k}| z_{i}\right), \\
& f_{3}(\mathbf{k})=\sum_{i=1}^{N} q_{i} \cos \left(k_{x} x_{i}+k_{y} y_{i}\right) \exp \left(+|\mathbf{k}| z_{i}\right),
\end{aligned}
$$

and

$$
f_{4}(\mathbf{k})=\sum_{i=1}^{N} q_{i} \sin \left(k_{x} x_{i}+k_{y} y_{i}\right) \exp \left(+|\mathbf{k}| z_{i}\right) .
$$

All functions need to be updated for each new configuration in the simulation. The method was developed in Ref. 46, 
TABLE I. The five sets of parameters considered in this work.

\begin{tabular}{lcc}
\hline \hline Sets & $\bar{a}_{H}$ & $\gamma$ \\
\hline Set 0 & 0.137 & 0 \\
Set 1 & 0.068 & 0.95 \\
Set 2 & 0.137 & 0.95 \\
Set 3 & 0.206 & 0.95 \\
Set 4 & 0.137 & -1 \\
\hline \hline
\end{tabular}

where it was tested against a previously developed method for low dielectric surfaces. ${ }^{45}$

The pressure between the surfaces is given by

$$
\beta P=\rho_{m}+F_{h} / A,
$$

where $\rho_{m}$ is the counterion density at the mid plane $z=L / 2$ and $F_{h}$ is the electrostatic force between all charges located in each half space $z>L / 2$ and $z<L / 2$. The pressure is defined to be positive and negative for repulsion and attraction, respectively. We define the rescaled pressure as

$$
\bar{P}=\frac{\beta P}{2 \pi \lambda_{B} \sigma^{2}} .
$$

In most simulations, we consider five different sets of parameters that are defined in Table I, and for each set, we vary the separation between the plates and the coupling parameter.

\section{RESULTS}

In Fig. 2(a), we show the density profiles of counterions for high coupling parameter $\Xi=1000$ and $\bar{a}_{H}=0.068$ for various values of $\gamma$. We observe that in the absence of dielectric boundaries, i.e., for $\gamma=0$, the counterion density profile is flat and well described by strong-coupling theory. ${ }^{32}$ For lowdielectric surfaces, for $\gamma=0.95$, the counterions are repelled from the surfaces, while for metallic surfaces, $\gamma=-1$, the counterions are strongly attracted to the surfaces, as expected due to image-charge interactions. Strong-coupling theory which uses the single-image charge approximation works very well for the high coupling parameter $\Xi=1000$, as demonstrated by the solid lines in Fig. 2(a), even for the metallic surfaces. ${ }^{32}$ The full and empty symbols represent the density profiles obtained by $\mathrm{MC}$ simulations using the first image approximation, simulated with the Lekner summation method, as was done in Ref. 32, and using the Green's function method which correctly includes all image charges ${ }^{46}$ respectively. The results show that the first image approximation works very well for low dielectric surfaces. Surprisingly, for metallic surfaces, the density profiles do not match. So we conclude that the singleimage approximation works fine for low-dielectric surfaces, but not for high-dielectric (metallic) surfaces. In Fig. 2(b), we compare pressure profiles for different dielectric contrast parameters $\gamma$ and two different coupling parameters $\Xi$. The curves are very similar for the low coupling parameter $\Xi=11$, while for high coupling $\Xi=1000$ significant deviations are observed.

Pressure curves for parameters corresponding to set 1 are shown in Fig. 3(a) as a function of the rescaled surface separation for various coupling parameters $\Xi$. In agreement
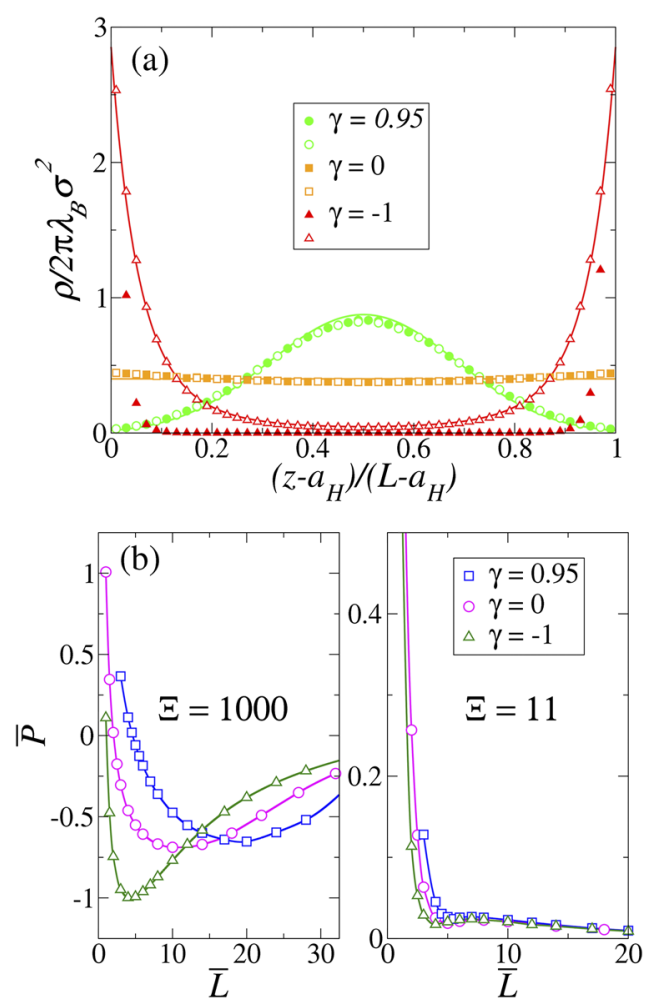

FIG. 2. (a) Counterion density profiles for $\bar{a}_{H}=0.068$ and for various values of $\gamma$ as indicated. The coupling parameter is $\Xi=1000$, while the distance between the surfaces is $\bar{L}=5$. The open symbols represent the present simulation method which includes all image charges, while full symbols represent the first image approximation used in earlier work. ${ }^{32}$ The solid lines correspond to the prediction of strong-coupling theory. ${ }^{32}$ (b) Pressure curves for $\bar{a}_{H}=0.137$ and two different coupling parameters $\Xi$ and different values of $\gamma$ as indicated in the figure. The solid lines are guides to the eye.

with previous observations, for high coupling parameter and for intermediate surface separation, the surfaces attract each other. In order to construct a thermodynamic phase diagram, we determine the free energy $\Phi(\bar{L})$ by integration over the pressure profile

$$
\Phi(\bar{L})=-\int_{\bar{L}}^{\bar{L}_{\infty}} \bar{P}\left(\bar{L}^{\prime}\right) d \bar{L}^{\prime}
$$

The free energy is defined to be zero at large separation $\bar{L}_{\infty}$, which is set to $\bar{L}_{\infty}=33$. This value is not sufficient to obtain the correct height of the free energy profile for $\Xi=1000$ in Fig. 3(b), but this is irrelevant for the calculation of the phase-coexistence region which only occurs for intermediate values of $\Xi$. In Fig. 3(b), the free energy profiles are shown for the same parameters as in Fig. 3(a). A coexistence region is discerned for intermediate values of coupling parameters in the inset, in which case a double-tangent construction can be done.

The pressure phase diagrams in Fig. 4 for different sets defined in Table I show the region, in which the pressure is negative and the charged surfaces attract each other. The attraction and repulsion regions are separated by stable (full symbols and solid lines) or metastable (empty symbols and dashed lines) lines on which the pressure is zero. The stability or instability on these lines is obtained by consideration of the free energy profiles shown in Fig. 3(b). 

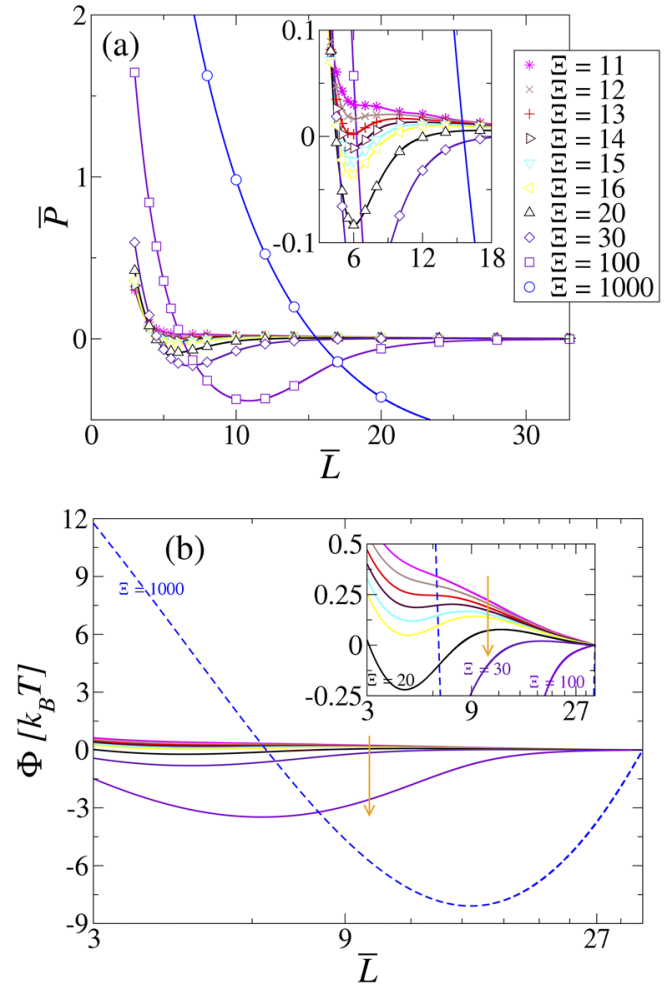

FIG. 3. (a) Pressure $\bar{P}$ as a function of the surface separation $\bar{L}$ for parameters defined in set 1 and for different coupling parameters $\Xi$ given in the legend. The lines are interpolation curves and the inset is the amplification. (b) Free energy $\Phi$ as a function of $\bar{L}$ for parameters defined in set 1 obtained by integration of the interpolated pressures in (a). The arrows indicate increasing values of coupling parameters, $\Xi=11,12,13,14,15,16,20,30,100$, and 1000. The inset is the amplification.

In Fig. 4(a), we show the pressure phase diagrams for parameter sets 1,2 , and 3, in which the dielectric parameter $\gamma$ is set to 0.95 (corresponding to low dielectric surfaces) and only the hydration length $\bar{a}_{H}$ changes; see Table I. For a given coupling parameter $\Xi$, for larger rescaled hydration length, the pressure is lowered and the region in which the pressure is negative increases. The limit of infinite hydration length corresponds to the case of no dielectric boundary. So we conclude that for low-dielectric surfaces, the regime of attraction is reduced, in agreement with previous results for $\gamma=0.95$ using the single-image approximation. ${ }^{32}$ In Fig. 4(b), pressure phase diagrams for sets 2,0 , and 4 are presented, where the hydration length is fixed and the dielectric contrast $\gamma$ is varied. For $\gamma=0.95$ (low dielectric surfaces), the attractive region is reduced, and for $\gamma=-1$ (metallic surfaces) it is increased compared to the case of vanishing dielectric contrast $\gamma=0$.

For a small range in coupling parameters between $\Xi=11$ and $\Xi=16$, a Maxwell construction can be used on the pressure curves to obtain the 2-phase coexistence region. ${ }^{32}$ This is exemplarily shown in Fig. 5 for set 1 and coupling constant $\Xi=13$.

The binodal curves, consisting of the coexistence plate separations, and the critical points are shown in Fig. 6 for four different parameter sets. As the coexistence pressure approaches zero, the large-distance branch of the binodal moves to infinite separation, which corresponds to an
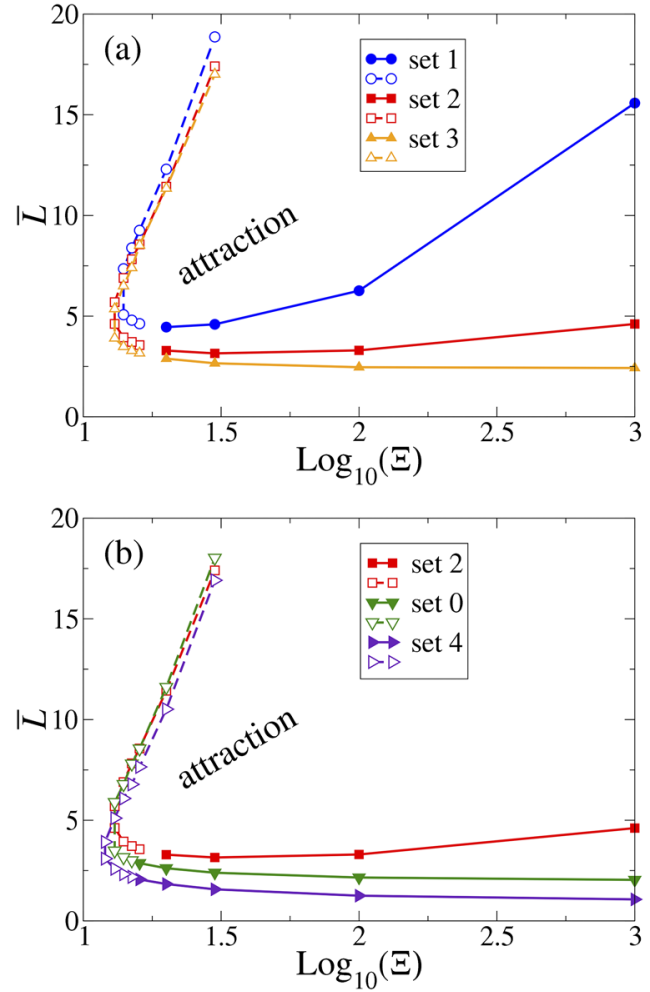

FIG. 4. Pressure phase diagrams for different sets of parameters. The lines are guide to the eye. The full and empty symbols represent stable and metastable lines on which the pressure vanishes, respectively. (a) The sets correspond to different values of the hydration parameter $\bar{a}_{H}$ for low dielectric surfaces with $\gamma=0.95$. (b) The sets correspond to different values of the dielectric contrast parameter $\gamma$, low-dielectric surfaces $\epsilon_{o}=\epsilon_{w} / 40(\gamma=0.95)$, no dielectric contrast $\epsilon_{o}=\epsilon_{w}(\gamma=0)$, and metallic surfaces, $\epsilon_{o}=\infty(\gamma=-1)$, for a fixed parameter $\bar{a}_{H}$.

unbinding transition between the surfaces. ${ }^{33}$ The coupling parameter at which this unbinding transition occurs is obtained by extrapolation of the coexistence pressure curves to $\bar{P}_{2}=0$, as shown in the insets of Fig. 6. We see in Fig. 6(a) that the coexistence curves and the critical points only slightly differ between sets 1 and 2, which means that the thermodynamic phase behavior depends very little on the hydration length. The effect of the dielectric mismatch on the coexistence curve can be understood by comparing parameter sets

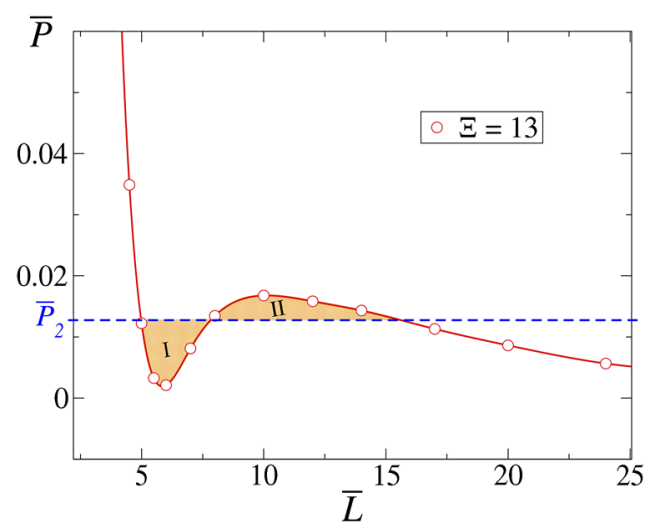

FIG. 5. Maxwell construction to determine the coexistence pressure $\bar{P}_{2}$ for the pressure profile obtained for set 1 and coupling parameter $\Xi=13$. The shaded areas I and II have the same area. 

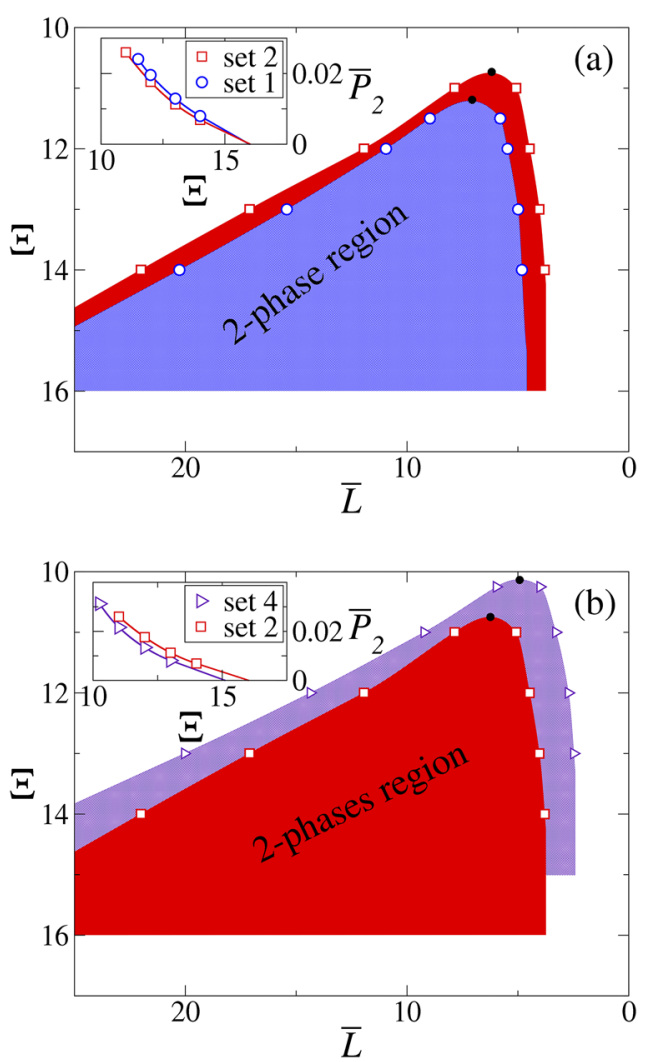

FIG. 6. Binodal curves for a few selected sets of parameters. The insets show the coexistence pressure $\bar{P}_{2}$ curve as a function of the coupling parameter $\Xi$. The shaded areas represent the 2-phase region. (a) Results for identical dielectric contrast parameters $\gamma$ and different values of the hydration length $\bar{a}_{H}$. The critical points are located at $\Xi \approx 11.2$ and $\Xi \approx 10.75$, for data sets 1 and 2 , respectively, represented by the full circles. The coupling parameter value at which the unbinding transition occurs is approximated by extrapolation, $\Xi \approx 16$, for both sets. (b) Results for the same hydration length $\bar{a}_{H}$ and two different values of $\gamma$. The critical points are located at $\Xi \approx 10.75$ and $\Xi \approx 10.13$, for data sets 2 and 4 , respectively, represented by the full circles. The coupling parameter at which the unbinding transition occurs is by extrapolation obtained as $\Xi \approx 15$ for set 4 .

2 and 4 in Fig. 6(b), which correspond to low-dielectric and high-dielectric surfaces. The difference is again very small. In fact, the critical points for all four systems shown in Fig. 6 are located at coupling constants very close to the value $\Xi \approx 10.25$ obtained previously for vanishing dielectric contrast. ${ }^{33}$ In previous simulation work, it was suggested ${ }^{33}$ that boundary dielectric effects could be an explanation for the deviation between the critical coupling constant found in simulations without dielectric contrast and in experiments. ${ }^{13,16}$ The present results show that dielectric effects shift the critical point characterizing the electrostatic surface adhesion between charged plates very slightly and thus cannot be the cause of the deviation between experiment and simulation. This deviation therefore must have to do with other effects that are not contained in our idealized model.

It is interesting to consider the effect of dispersion interactions on our prediction which arise from fluctuations in the electrostatic potential. ${ }^{49,50}$ The van der Waals pressure contribution $\beta P_{v}=\mathrm{A} / 8 \pi L^{3}$ can be written in a rescaled form as $^{49}$

$$
\bar{P}_{v}=\frac{\mathrm{A} \Xi}{4\left(\bar{L}+2 \bar{a}_{H} \sqrt{\Xi}\right)^{3}},
$$
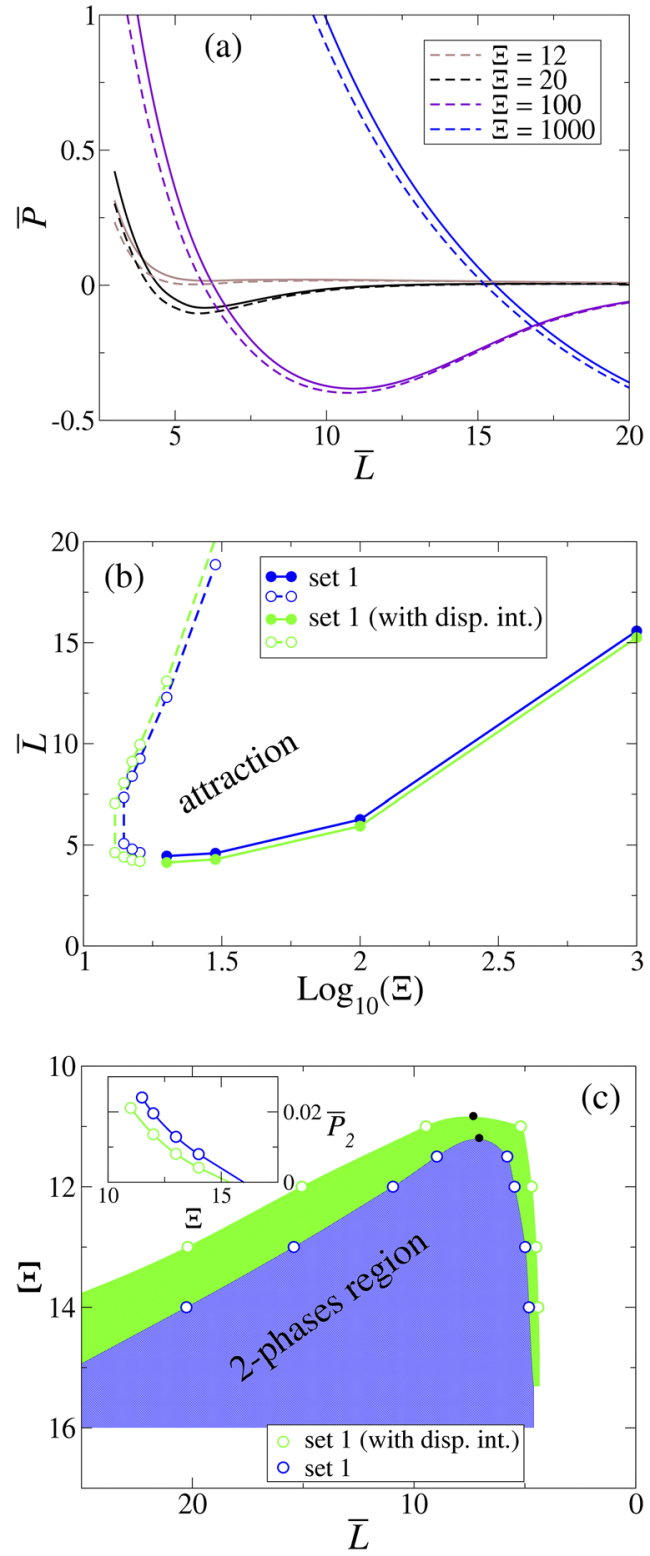

FIG. 7. (a) Pressure $\bar{P}$ as a function of the surface separation $\bar{L}$ for parameters defined in set 1 and for different coupling parameters given in the legend. The solid and dashed lines represent the cases without and with the addition of dispersion interactions defined in Eq. (14), respectively. (b) Pressure phase diagrams and (c) binodals for set 1 without and with the addition of dispersion interactions, represented by blue and green curves, respectively. The critical point for set 1 with dispersion interaction is shifted from $\Xi_{c} \approx 11.2$ to $\Xi_{c} \approx 10.9$.

where A is the Hamaker constant,

$$
\mathrm{A}=\int_{0}^{\infty} d p p \ln \left(1-\gamma^{2} e^{-p}\right) .
$$

In Fig. 7, we compare the pressures, pressure phase diagrams, and binodals obtained with and without the van der Waals contribution, Eq. (14). It can be observed that the dispersion contribution only slightly changes the results.

\section{CONCLUSIONS}

We have obtained pressure phase diagrams and coexistence curves for two interacting equally charged surfaces in the presence of point-like counterions. Exclusion zones near the 
surfaces are considered which model ionic surface hydration effects in the form of a steric interaction between the surface and counterions. We observe that although the counterion density profiles depend sensitively on the dielectric contrast at the surfaces and on the hydration length, the resulting pressure profiles, the pressure phase diagrams, and in particular the coexistence curves around the critical point do not depend significantly on these parameters and on dispersion interactions. Only for high coupling parameters, the pressure curves change considerably. The first-image approximation works very well for low-dielectric surfaces; however, its numerical implementation relies on the Lekner summation, and thus, there is no numerical benefit compared to the exact periodic Green's function method employed in this work. For metallic surfaces, the counterion density profiles obtained from the first-image approximation and Green's function method deviate strongly from each other. Strong-coupling theory is shown to work very well for high coupling parameters and for both low-dielectric and metallic surfaces.

\section{ACKNOWLEDGMENTS}

This work was supported by the Alexander von Humboldt Foundation through the CAPES program and by the European Innovative Training Network "Transport of Soft Matter at the Nanoscale" (NANOTRANS).

${ }^{1}$ C. A. Keller, K. Glasmastar, V. P. Zhdanov, and B. Kasemo, Phys. Rev. Lett. 84, 5443 (2000).

${ }^{2}$ R. P. Richter and A. R. Brisson, Biophys. J. 88, 3422 (2005).

${ }^{3}$ K. Funakoshi, H. Suzuki, and S. Takeuchi, Anal. Chem. 78, 8169 (2006).

${ }^{4}$ N. Malmstadt, M. A. Nash, R. F. Purnell, and J. J. Schmidt, Nano Lett. 6, 1961 (2006).

${ }^{5}$ S. R. Tabaei, W. B. Ng, S. J. Cho, and N. J. Cho, ACS Appl. Mater. Interfaces 8, 11875 (2016).

${ }^{6}$ L. Illum and S. S. Davis, Life Sci. 40, 1553 (1987).

${ }^{7}$ D. Needham, T. J. McIntosh, and D. D. Lasic, Biochim. Biophys. Acta 1108, 40 (1992).

${ }^{8}$ K. Epler, D. P. G. Phillips, P. Crowder, R. Castillo, D. Wilkinson, B. Wilkinson, C. Burgard, R. Kalinich, J. Townson, B. Chackerian, C. Willman, D. Peabody, W. Wharton, C. J. Brinker, C. Ashley, and E. Carnes, Adv. Healthcare Mater. 1, 348 (2012).

${ }^{9}$ C. G. Siontorou, G. P. Nikoleli, D. P. Nikolelis, and S. K. Karapetis, Membranes 7, 38 (2017).

${ }^{10}$ E. Sackmann, Science 271, 43 (1996).

${ }^{11}$ J. N. Israelachvili and H. Wennerström, J. Phys. Chem. 96, 520 (1992).

${ }^{12}$ M. Dubois, T. Zemb, and L. Belloni, J. Chem. Phys. 96, 2278 (1992).

${ }^{13}$ T. Zemb, D. Gazeau, M. Dubois, and T. Gulik-Krzywicki, Europhys. Lett. 21, 759 (1993).

${ }^{14}$ L. L. Brasher, K. L. Herrington, and E. W. Kaler, Langmuir 11, 4267-4277 (1995).
${ }^{15}$ S. Chiruvolu, J. N. Israelachvili, E. Naranjo, Z. Xu, J. A. Zasadzinski, E. W. Kaler, and K. L. Herrington, Langmuir 11, 4256-4266 (1995).

${ }^{16}$ M. Dubois, T. Zemb, N. Fuller, R. P. Rand, and V. A. Parsegian, J. Chem. Phys. 108, 7855 (1998).

${ }^{17}$ H. Butt, B. Cappella, and M. Kappl, Surf. Sci. Rep. 59, 1 (2005).

${ }^{18}$ B. V. Derjaguin and L. Landau, Acta Physicochim. URSS 14, 633 (1941).

${ }^{19}$ E. J. W. Verwey and J. T. G. Overbeek, Theory of the Stability of Lyophobic Colloids (Elsevier, Amsterdam, 1948).

${ }^{20}$ Y. Levin, Rep. Prog. Phys. 65, 1577 (2002).

${ }^{21}$ M. Kanduc, A. Schlaich, E. Schneck, and R. R. Netz, Langmuir 32, 8767 (2016).

${ }^{22}$ B. Kowalik, A. Schlaich, M. Kanduc, E. Schneck, and R. R. Netz, J. Phys. Chem. Lett. 8, 2869-2874 (2017).

${ }^{23}$ P. Kékicheff, S. Marčelja, T. J. Senden, and V. E. Shubin, J. Chem. Phys. 99, 6098 (1993).

${ }^{24}$ T. Keszthelyi, G. Hollo, G. Nyitrai, J. Kardos, and L. Heja, Langmuir 31, 7815 (2015).

${ }^{25}$ H. I. Petrache, T. Zemb, L. Belloni, and V. A. Parsegian, Proc. Natl. Acad. Sci. U. S. A. 103, 7982 (2006).

${ }^{26}$ A. P. dos Santos and Y. Levin, Phys. Rev. Lett. 106, 167801 (2011).

${ }^{27}$ M. L. Berkowitz and R. Vacha, Acc. Chem. Res. 45, 74 (2012).

${ }^{28}$ R. Kjellander, T. Åkesson, B. Jönsson, and S. Marčelja, J. Chem. Phys. 97, 1424 (1992).

${ }^{29}$ I. Rouzina and V. A. Bloomfield, J. Phys. Chem. 100, 9977-9989 (1996).

${ }^{30}$ R. R. Netz, Eur. Phys. J. E 5, 557 (2001).

${ }^{31}$ A. G. Moreira and R. R. Netz, Eur. Phys. J. E 8, 33 (2002).

${ }^{32}$ A. Naji, S. Jungblut, A. G. Moreira, and R. R. Netz, Phys. A 352, 131 (2005).

${ }^{33}$ H. Boroudjerdi, Y. W. Kim, A. Naji, R. R. Netz, X. Schlagberger, and A. Serr, Phys. Rep. 416, 129 (2005).

${ }^{34}$ Y. S. Jho, M. Kanduc, A. Naji, R. Podgornik, M. W. Kim, and P. A. Pincus, Phys. Rev. Lett. 101, 188101 (2008).

${ }^{35}$ D. F. Parsons, M. Bostrom, P. Lo Nostro, and B. W. Ninham, Phys. Chem. Chem. Phys. 13, 12352 (2011).

${ }^{36}$ L. Samaj and E. Trizac, Europhys. Lett. 100, 56005 (2012).

${ }^{37}$ J. W. Zwanikken and M. O. de la Cruz, Proc. Natl. Acad. Sci. U. S. A. 110, 5301 (2013).

${ }^{38}$ R. Wang and Z. G. Wang, J. Chem. Phys. 139, 124702 (2013).

${ }^{39}$ S. Buyukdagli, M. Manghi, and J. Palmeri, Phys. Rev. E 81, 041601 (2010).

${ }^{40}$ Y. S. Jho, G. Park, C. S. Chang, P. A. Pincus, and M. Kim, Phys. Rev. E 76, 011920 (2007).

${ }^{41}$ S. Tyagi, A. Arnold, and C. Holm, J. Chem. Phys. 129, 204102 (2008).

${ }^{42}$ M. Kanduc, A. Naji, J. Forsman, and R. Podgornik, J. Chem. Phys. 137, 174704 (2012).

${ }^{43}$ V. Jadhao, F. J. Solis, and M. O. de la Cruz, Phys. Rev. Lett. 109, 223905 (2012).

${ }^{44}$ C. Berti, S. Furini, D. Gillespie, D. Boda, R. S. Eisenberg, E. Sangiorgi, and C. Fiegna, J. Chem. Theory Comput. 10, 2911 (2014).

${ }^{45}$ A. P. dos Santos and Y. Levin, J. Chem. Phys. 142, 194104 (2015).

${ }^{46}$ A. P. dos Santos, M. Girotto, and Y. Levin, J. Chem. Phys. 147, 184105 (2017).

${ }^{47}$ A. P. dos Santos, M. Girotto, and Y. Levin, J. Chem. Phys. 144, 144103 (2016).

${ }^{48}$ R. Wang and Z. Wang, J. Chem. Phys. 142, 104705 (2015).

${ }^{49}$ R. R. Netz, Eur. Phys. J. E 5, 189 (2001).

${ }^{50}$ D. S. Dean and R. R. Horgan, Phys. Rev. E 69, 061603 (2004). 